\title{
Synthesis of Uniform and Stable Molecularly Imprinted Polymer Particles by Precipitation Polymerization
}

\author{
RACHANEEWAN KARNKA ${ }^{1 *}$, PREEYAPORN CHAIYASAT ${ }^{2,3}$, \\ and AMORN CHAIYASAT ${ }^{2,3 * *}$ \\ 'Department of Applied Chemistry, , Program in Applied Chemistry, Faculty of Sciences \\ and Liberal Arts, Rajamangala University of Technology Isan, Nakhon \\ Ratchasima 30000, Thailand. \\ 2Department of Chemistry, Faculty of Science and Technology, Rajamangala University of \\ Technology Thanyaburi, Klong 6, Thanyaburi, Pathumthani 12110, Thailand. \\ ${ }^{3}$ Advanced Materials Design and Development (AMDD) Research Unit, Institute of \\ Research and Development, Rajamangala University of Technology Thanyaburi, \\ Klong 6,Thanyaburi, Pathumthani 12110, Thailand. \\ *Corresponding author E-mail: Rachaneewan.ka@ rmuti.ac.th, a_chaiyasat@mail.rmutt.ac.th
}

http://dx.doi.org/10.13005/ojc/330529

(Received: May 10, 2017; Accepted: July 20, 2017)

\section{ABSTRACT}

In this work, precipitation polymerization was used to prepare uniform and stable molecular imprinting polymers (MIPs) particles without post treatment. Melamine was used as a template molecule model, while methacrylic acid, divinyl benzene (DVB) or ethylene glycol dimethacrylate (EGDMA) and acetonitrile were used as functional monomer, cross-linker and continuous phase, respectively. The polymerization was carried out in a vacuum condition at $70^{\circ} \mathrm{C}$ using benzoyl peroxide as an initiator. The obtained MIP particles demonstrated spherical shape with narrow particle size distribution. The particle size varied from micrometer-sized to sub-micrometer-sized depending on the initiator content. After purification, MIP particles represented high colloidal stability in aqueous medium. The binding efficiency of MIP using both cross-linkers (DVB and EGDMA) with melamine was approximately $20 \mathrm{mg} / \mathrm{g}$ MIP. The miscibility of all components where the functional monomer effectively pre-bound with the template molecules was the main factor for obtaining MIP particle with high binding efficiency.

Keywords: Uniform MIP particle, Melamine, Precipitation polymerization, Homogeneous nucleation.

\section{INTRODUCTION}

Molecular imprinting polymers (MIPs) are known as facile and versatile materials for the creation of tailor-made binding sites which memorized template molecules with shape, size and functional groups ${ }^{1}$. To produce the durable MIPs, the cross-linked MIPs are synthesized by copolymerization of functional monomer (pre-bound with template molecule) and crosslink monomer ${ }^{2,3}$. MIPs are able to selectively rebind the target molecules from the sample when the template 
molecules are removed giving recognition cavity matching to the template molecule in shape, size and chemical functionality formed in polymer matrix. Due to numerous advantages of MIPs as good mechanical, thermal, and chemical stabilities with high selectivity to the target molecules, they are extremely practical in a wide range of applications such as selective stationary phase for solid-phase extraction (SPE) $)^{4,5}$, chromatographic separation ${ }^{6,7}$, chemical sensors ${ }^{8}$ and drug controlled release ${ }^{9,10}$. Generally, there are two binding interactions between template and functional monomer, one employing covalent bond and the other utilizing non-covalent interactions. In the case of covalent linkage, templates suitable for covalent imprinting are limited because MIPs require identical rebinding linkages between target molecules and functional monomers. Moreover, MIPs require strong interaction, it is very difficult to reach thermodynamic equilibrium ${ }^{3}$. In contrast, noncovalent imprinting having various binding interactions such as hydrogen bonding and van der waals forces is more flexible. Furthermore, it is simple to prepare MIPs which require only mixing of templates and monomers in suitable conditions. Therefore, non-covalent imprinting has currently turned into the most famous and useful synthesis for MIPs preparation. Several polymerization processes such as homogeneous (bulk polymerization ${ }^{11}$ ) and heterogeneous systems such as suspension ${ }^{12}$, miniemulsion ${ }^{13}$, seeded emulsion ${ }^{14}$, emulsion ${ }^{15}$ and precipitation ${ }^{5,9,10,16,17}$ polymerizations are used to prepare MIPs. Bulk polymerization is firstly used to prepare MIPs. It is a simple technique which gives high purity of MIP products. However, the main drawback of bulk polymerization is the requirement of the post treatment step as grinding and sieving MIPs to obtain the appropriate size matching with the application. As a result, more time is needed and loss some MIPs yield could happen. Moreover, some affinity binding sites cause physical damage during grinding process. To overcome these drawbacks, polymerization in heterogeneous system would be more appropriate to produce the MIPs particles. However, most of them (suspension, dispersion, seeded, miniemulsion and emulsion polymerizations) use emulsifier in the process which is adsorbed on the surface of the obtained particles. Thus, emulsifiers would disturb the desired recognition and selective binding of target molecules. Therefore, the polymerization process without emulsifier is an interesting and suitable method to produce MIPs particles. The MIPs particles are formed by homogeneous nucleation in the precipitation polymerization. All components are firstly miscible. After polymerization, the grown polymer chains reach their critical chain length and are unable to dissolve in the continuous phase. Thus, they self-assemble to form the particle and precipitate out. Although numerous of the MIPs preparations were reported with various cross-linker monomers such as divinylbenzene (DVB), ethylene glycol dimethacrylate (EGDMA) and trimethylolpropane trimethacrylate (TRIM), the influence of the cross-linker type, in which may affect the miscibility of pre-polymerization solution and on the binding efficiency of MIPs has not been reported. Therefore, in this work, MIPs particles were prepared by precipitation polymerization using methacrylic acid (MAA) as a functional monomer and melamine as template molecule. The influences of cross-linker, initiator and template molecule amounts on the binding property were studied.

\section{EXPERIMENTAL}

\section{Materials}

DVB (Aldrich, Wisconsin, USA; purity, 80\%) was washed with $1 \mathrm{M}$ sodium hydroxide $(\mathrm{NaOH}$; BDH Prolabo, Leuven, Belgium) solution and distilled water to remove polymerization inhibitors before use. Methacrylic acid (MAA) (Merck \& Co., Munich, Germany; purity, 99\%) was recrystallized in frozen water. Ethyleneglycoldimethacrylate (EGDMA; Aldrich, Wisconsin, USA; purity, 99\%)and trimethylolpropanetrimethacrylate (TRIM; Aldrich, Wisconsin, USA; purity, 90\%) were purified by passing them through a column packed with basic aluminum oxide to remove inhibitor. The purified monomers were stored in a refrigerator. Acetic acid (RCI Labscan, Bangkok, Thailand; 96\%), methanol $(\mathrm{MeOH})(\mathrm{RCl}$ Labscan, Bangkok, Thailand; purity, 99.9\%), ethanol (EtOH) (RCl Labscan, Bangkok, Thailand; $99.9 \%)$ and acetonitrile (ACN)(RCI Labscan, Bangkok, Thailand; 99.7\%) were used as received. Reagent-grade benzoyl peroxide (BPO; Merck \& Co., Munich, Germany) was dissolved in chloroform before recrystallizing in methanol. 


\section{NIP/MIP particles preparation}

The MIP particles were prepared by precipitation polymerization under the conditions listed in Table 1. Individually, preparation of the accurate amount of functional monomer as a MAA was mixed with accurate amount of the template (target molecule)as a melamine using sonication until completely miscible while accurate amount of the cross-linker as a DVB or EGDMA or TRIM was completely dissolved in the continuous phase of acetonitrile. There after, both solutions were homogeneously mixed using mind stirring rate before pouring into a round bottom flask sealed with silicone rubber septum. The solution was purged with five vacuum $/ \mathrm{N}_{2}$ cycles finally in a $\mathrm{N}_{2}$ atmosphere. The polymerization was started with the injection of BPO solution in acetonitrile. It was then polymerized at $70^{\circ} \mathrm{C}$ for $6 \mathrm{~h}$ at a stirring rate of $200 \mathrm{rpm}$. The resultant MIPs particles were separated from the reaction mixture by centrifugation. Finally, the particles were dried in a vacuum at $40^{\circ} \mathrm{C}$ to obtain constant weight. Nonmolecular imprinting polymers (NIPs) were also prepared in the same manner without the template as control experiment.

\section{Characterizations}

Percent monomer conversion was measured by gravimetry. The prepared NIP/MIP particles were observed with an optical microscope
(OM) (SK-100EB \& SK-100ET, Seek, Seek Inter Co. Ltd., Thailand) and scanning electron microscope (SEM) (JSM-6510, JEOL, JEOL Ltd., Japan) to study the morphology of the particle surface and shape. For SEM observations, one drop of the polymer suspension was placed on a nickel SEM stub and dried before being coated with Au. Average volume and average number of diameters $\left(d_{v}\right.$ and $d_{n}$ respectively) were measured by dynamic light scattering (DLS, Delsa Nano C, Beckman Coulter, Germany) at the light scattering angle of $165^{\circ} \mathrm{C}$ at room temperature. The MIPs particlesdispersed in water (approximately $10 \mathrm{wt} \%$ ) were measured with the concentration mode.The binding efficiency (BE) of the MIPs and NIPs particles were evaluated by equilibrium binding experiments. Firstly, the template and residual monomer/oligomer were extracted by dissolving the dried MIPs or NIPs particles $(0.50 \mathrm{~g})$ in $10 \mathrm{ml}$ of $5 \mathrm{wt} \%$ of acetic acid in methanol for overnight. The polymer particles were then dispersed in $10 \mathrm{ml}$ of methanol for $2 \mathrm{~h}$. two times. To confirm complete removal of all residue template from the MIPs particles, the amount of template in the washed methanol was measured by a high performance liquid chromatograph (HPLC; Dionex, CA, USA) equipped with a C18 column (Dionex Acclaim $₫$ Polar Advantage 2, $3 \mu \mathrm{m}$ particle size, $120 \mathrm{~A}^{\circ}$ pores size, $2.1 \mathrm{~mm}$ i.d $\times 150 \mathrm{~mm}$ long, Dionex, CA, USA). Thus, $20 \mu \mathrm{l}$ of the washed methanol was injected

Table. 1: Recipes for the preparation of non-molecular imprinting and molecular imprinting polymers particles by precipitation polymerization.

\begin{tabular}{lcccccc}
\hline Experiment & MAA(mmol) & DVB $(\mathrm{mmol})$ & EGDMA $(\mathrm{mmol})$ & BPO $(\mathrm{mmol})$ & Melamine $(\mathrm{mmol})$ & ACN (g) \\
\hline 1 & 5.00 & - & 1.00 & 1.00 & 0.12 & 47.00 \\
2 & 10.00 & - & 1.00 & 1.00 & 0.12 & 47.00 \\
3 & 20.00 & - & 1.00 & 1.00 & 0.12 & 47.00 \\
4 & 20.00 & - & 1.00 & 2.00 & 0.12 & 47.00 \\
5 & 20.00 & - & 1.00 & 3.00 & 0.12 & 47.00 \\
6 & 20.00 & - & 1.00 & 3.00 & - & 47.00 \\
7 & 20.00 & 1.00 & - & 3.00 & 0.12 & 47.00 \\
8 & 20.00 & 1.00 & - & 3.00 & - & 47.00 \\
9 & 20.00 & 1.00 & - & 3.00 & 0.25 & 47.00 \\
10 & 20.00 & 1.00 & - & 3.00 & 0.50 & 47.00 \\
11 & 20.00 & - & 1.00 & 3.00 & 0.25 & 47.00 \\
12 & 20.00 & - & 1.00 & 3.00 & 0.50 & 47.00 \\
\hline Abbra
\end{tabular}

Abbreviations:

MAA; methacrylic acid, DVB; divinyl benzene, EGDMA; Ethyleneglycol dimethacrylate, BPO; benzoylperoxide, CAN; acetonitrile. 
into the HPLC using methanol as the eluent at 0.2 $\mathrm{ml} / \mathrm{min}$ flow rate. The samples were detected with photodiode array detector at $215 \mathrm{~nm}$. Secondly, approximately $0.02 \mathrm{~g}$ of the washed MIPs and NIPs particles were individually immersed into $5 \mathrm{ml}$ of $100 \mathrm{mg} / \mathrm{l}$ of melamine solution in methanol and stirred with mild condition for $24 \mathrm{~h}$. at room temperature to ensure that they reached to equilibrium adsorption. The mixture was then centrifuged $(8,000 \mathrm{rpm}$ for $10 \mathrm{~min}$.$) and their$ supernatants were measured by HPLC at the same condition of washing step compared with melamine standard curve. The amount of melamine bound to MIPs or NIPs particles was calculated by subtraction of the amount of melamine in the solution after immersion from the initial amount as shown in the equation 1 and 2.

$\mathrm{BE}=\mathrm{W}_{\mathrm{B}} / \mathrm{W}_{\mathrm{MIP}}$

$\mathrm{W}_{\mathrm{B}}=\frac{\left[\mathrm{T}_{\text {initial }}\right]-\left[\mathrm{T}_{\text {sup }}\right]}{1000} \times \mathrm{V}_{\mathrm{S}}$

Where $B E$ is binding efficiency in the unit of $\mathrm{mg}$ of melamine to $\mathrm{g}$ of MIP/NIP particle, $\left[\mathrm{T}_{\text {initial }}\right]$ is the concentration $(100 \mathrm{mg} / \mathrm{l})$ of melamine before immersed by MIP/NIP particles, $\left[\mathrm{T}_{\text {sup }}\right]$ is the concentration $(\mathrm{mg} / \mathrm{l})$ of melamine in supernatant, $\mathrm{V}_{\mathrm{s}}$ is the volume $(5 \mathrm{ml})$ of melamine solution and $\mathrm{W}_{\text {MIP }}$ is mass $(0.02 \mathrm{mg})$ of the used MIP/NIP particles.

\section{RESULTS AND DISCUSSION}

In general, the mole ratio of functional monomer to cross-linker of the precipitation polymerization of MIP particles is always higher than $1^{12,18,19}$.Therefore, the first polymerization trial was started with 1:1 mole ratio of MAA and crosslinkers (EGDMA, DVB or TRIM). It was found that all components of all conditions were not completely miscible especially in the case of TRIM where most of MAA, TRIM and melamine seemed completely separated out from the continuous phase of acetonitrile in which precipitation polymerization was unable to proceed. This indicates that TRIM was not appropriate for synthesizing MIP particles in precipitation polymerization system at least in this work. In the case of EGDMA (Fig. 1a) and DVB (Fig. 1b), all components were partially soluble in acetonitrile in which a few MIP microparticle-sized was prepared with large amount of coalescence. After removal of particle aggregation, approximately 2-5 mm spherical MIP particles were observed in both cases as shown in Fig. 1a and b.Normally, MIP particles are formed by homogeneous nucleation in acetonitrile where polymerizing chains reach to their critical chain lengths and are unable to dissolve therein. Thus, they self-assemble to form the particle and precipitate out. However, the recognition of the obtained MIP particles of both cross-linkers were not observed where their BE were not different from those of NIP particles. This indicates that most of the melamine molecules partitioned in MAA and cross-linkers were difficult to diffuse to acetonitrile where the polymerization locus is. The important point for successfully preparing the stable MIPs particles containing the recognition cavity on their surfaces are that all components have to be completely miscible. Therefore, MAA can bind with melamine before polymerization. Therefore, the miscibility of all components was then studied with various MAA amounts as follow.
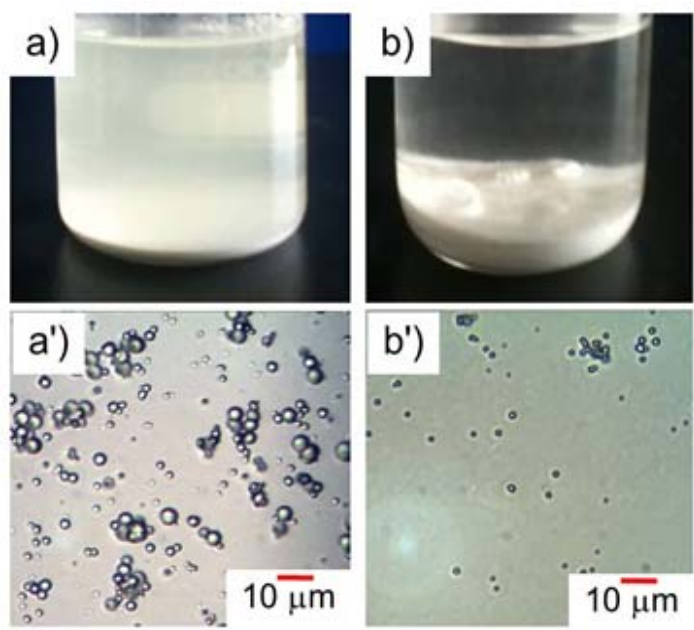

Fig. 1.Solution photos (a and b) before precipitation polymerization and optical micrographs (a' and b') of MIP particles (after removal of particle aggregation) using EGDMA (a and a') and DVB (b and b') as cross-linkers.

The solutions of various MAA contents are shown in Fig. 2 where the mole ratio of MAA to EGDMA is 5 (Fig. 2a), 10 (Fig. 2b) and 20 (Fig. 2c) times according to experiments 1-3 in Table 1. It was found that the miscibility of all components increased with MAA amount and completely miscible at 20 times of MAA to EGDMA as clear solution without precipitation in the bottom was observed. In the cases of 5 and 10 times of MAA to EGDMA, unstable MIP particles were still obtained 
similar to 1 time of MAA to EGDMA as representing large amount of particle coalescence. In the case of 20 times of MAA to EGDMA, stable micrometersized MIP particles without coagulation were obtained (Fig. 3a). Therefore, this condition was then selected for the preparation of MIP particles for further study.
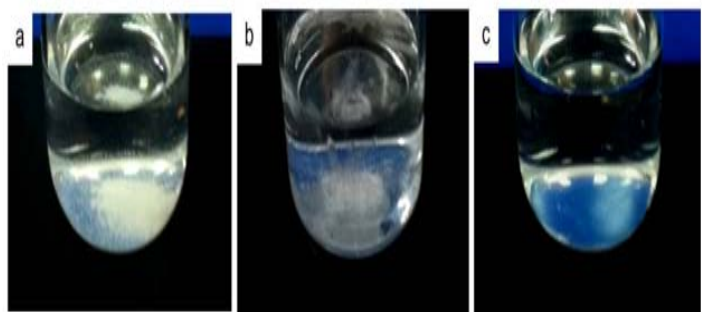

Fig. 2. Solution photos before precipitation polymerization at various mole ratios of MAA : EGDMA: 5 : 1 (a), 10:1 (b) and $20: 1$ (c) according to experiments 1-3 in Table 1
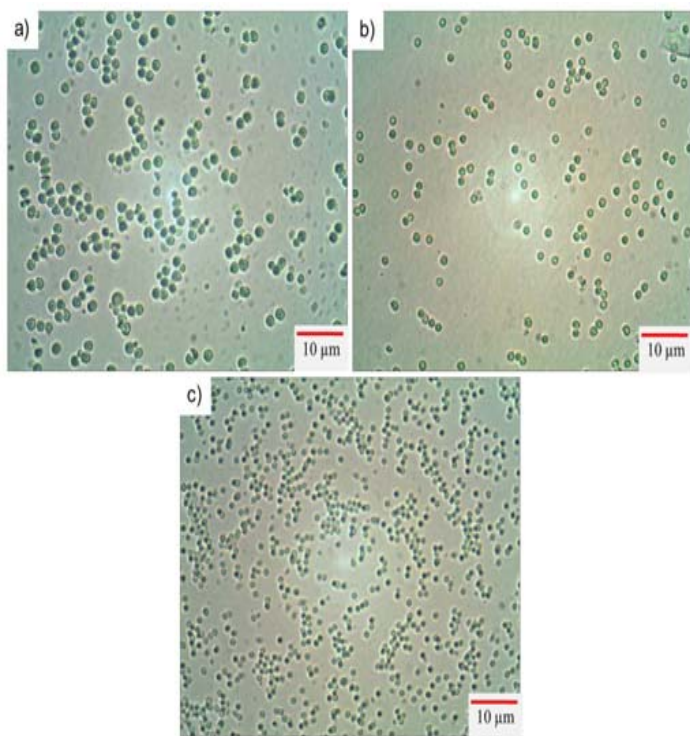

Fig. 3. Optical micrographs of MIP particles prepared by precipitation polymerization using MAA : EGDMA

(molar ratio) $=20: 1$ at various amounts of BPO (mmol) : (a) 1 (experiment 3), (b) 2 (experiment 4) and (c) 3 (experiment 5)

To reduce the polymerization time, the rate of polymerization was increased with increasing of initiator as BPO content from 1 to $3 \mathrm{mmol}$. Percent monomer conversions of the obtained MIP particle were 93,99 and $100 \%$ by $6 \mathrm{~h}$ for 1,2 and $3 \mathrm{mmol}$ of $\mathrm{BPO}$, respectively. In all cases, the obtained MIP particles were spherical without coalescence and could disperse in aqueous medium several times.
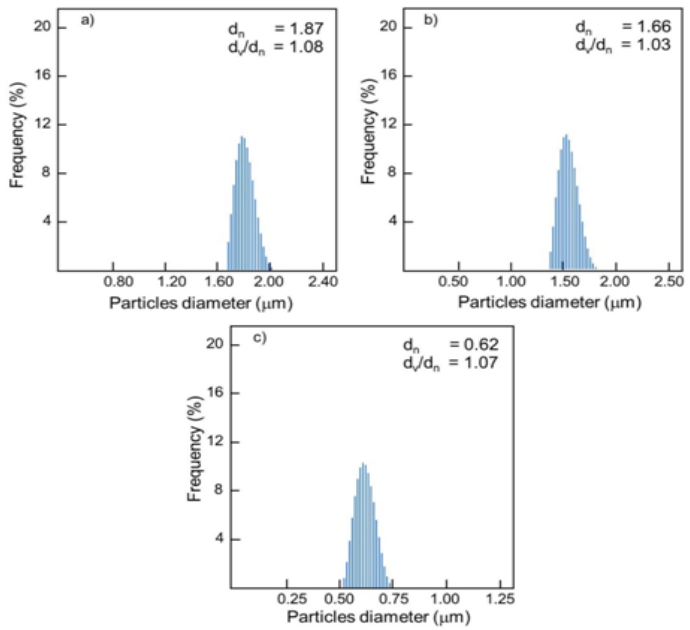

Fig.4. Particle size distributions (number distribution) (measured by DLS) of MIP particles prepared by precipitation polymerization using

MAA : EGDMA (molar ratio) $=20: 1$ at variousamounts of $B P O(\mathrm{mmol})$ : (a)1(experiment 3), (b) 2 (experiment 4) and (c) 3 (experiment 5).

When MIP particles were dispersed in water, the carboxyl group of MAA units in the polymer chain located on their particle surfaces might be deprotonated and effectively stabilized MIP particles. MIP particle size gradually decreased with BPO amount in which their average numbers of diameters were $1.87,1.66$ and $0.62 \mathrm{~mm}$ for 1,2 and $3 \mathrm{mmol}$ of BPO, respectively, as shown in Fig. 4. In addition, the MIP particle size distributions of all conditions were quite narrow as $d / d_{n}$ values were close to 1 . The decrease of particle size might be due to the increase of the number of polymerizing chain. Based on the smallest of the obtained MIP particles using $3 \mathrm{mmol}$ of BPO with polymerization time of $6 \mathrm{~h}$, it was then selected for further study.

Various kinds of cross-linker were used to synthesize MIP particles both hydrophobic and hydrophilic compounds as DVB ${ }^{20,21}$ and EGDMA ${ }^{22,23}$, respectively. Therefore, the influence of the kinds of cross-linker on the MIP properties was thus studied. Precipitation polymerization of MIP using $\mathrm{MAA} /$ cross-linker mole ratio of 20:1 with 3 and 0.12 $\mathrm{mmol}$ of BPO and malemine, respectively, was studied using EGDMA or DVB as a cross-linker. It was found that for both conditions, MIP particles were spherical in sub-micrometer sized without particle aggregation (Fig. $5 \mathrm{c}$ and $5 \mathrm{~d}$ ). The polarity 
of cross-linker seemed to have less effect on the size of MIP particles. It would be due to low amount of cross-linker related to MAA meanwhile the larger amount of MAA would be enough to stabilize MIP particles leading to the formation of a similar size. This result was also observed in the case of NIP particles (Fig. 5a and 5b). However, the difference in both cross-linkers was clearly observed for the polymerization rate. It was found that with the hydrophilic cross-linker as EGDMA, rate of polymerization ( $100 \%$ by $4 \mathrm{~h}$ ) was quite higher than that $(73 \%$ by $4 \mathrm{~h})$ of the hydrophobic cross-linker as DVB (Figure. 6).

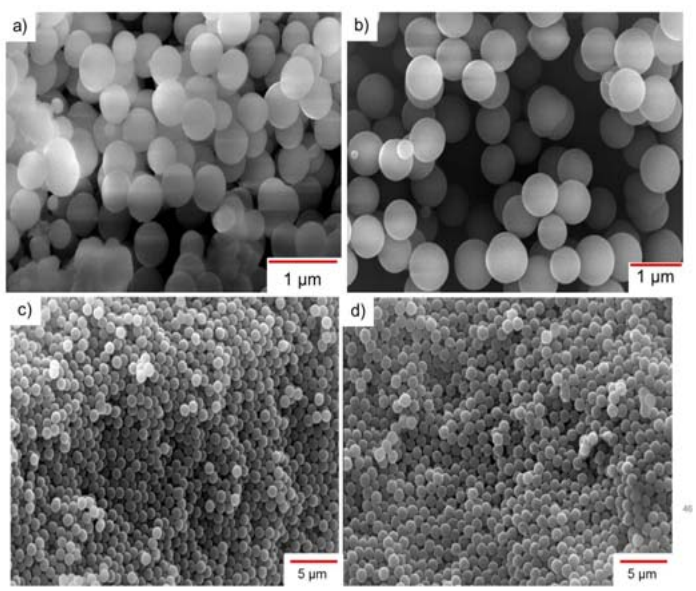

Fig. 5. SEM micrographsof NIP ( $a$ and $b$ ) and MIP particles ( $c$ and d) prepared by precipitation polymerization of MAA : cross-linker (molar ratio) $=\mathbf{2 0}$ : 1 with $3 \mathrm{mmol}$ of BPO using difference crosslinkers: EGDMA (a : experiment 6 and $c$ : experiment 5) and DVB (b : experiment 7and $d$ : experiment 8 ).

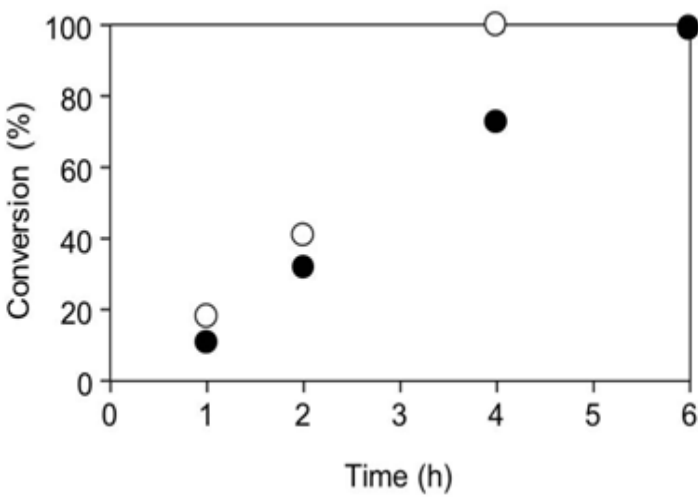

Fig. 6. Conversion-time curves of MIP particles with MAA : cross-linker (molar ratio) $=20: 1$ using difference crosslinkers : EGDMA (open circle ; experiment 5) and DVB (closed circle ; experiment 7).
Before study the BE of the obtained MIP particles, the template and residual monomer/ oligomer in the obtained MIP particles were extracted with $5 \%$ acetic acid in methanol and then washed with methanol until melamine peak in HPLC chromatogram was not found. Due to high content of MAA, the purified MIP particles could smoothly disperse in melamine standard aqueous solution. The melamine concentrations in aqueous solution both before and after immersion of MIP particles were determined by HPLC technique. The results in Fig. 7 shown that peak area of melamine in supernatant after removing MIP particles (using DVB as the cross-linker) (Fig. 7c) significantly reduced from the original one (Fig. 7a) where it seemed not different with NIP particles (Fig. 7b). Thereafter, the obtained results were used to calculate $\mathrm{BE}$ value with equation 1 and 2 . It was found that BE values of MIP particles with EGDMA (19 mg-melamine/g-MIP) and DVB (20 mgmelamine/g-MIP) as cross-linkers were not different. In addition, these data were much higher than their NIP particles (0.2 and $0.1 \mathrm{mg}$-melamine/g-NIP for EGDMA and DVB, respectively). This indicates that the obtained MIP particles of both cross-linkers represented high affinity to rebind the melamine template molecules. In addition, the kind of crosslinker was not significantly effective to bind efficiently; meanwhile the main factor was the functional monomer as MAA. To increase the binding efficiency, the amount of template molecule was also increased. However, it is unfortunate that further

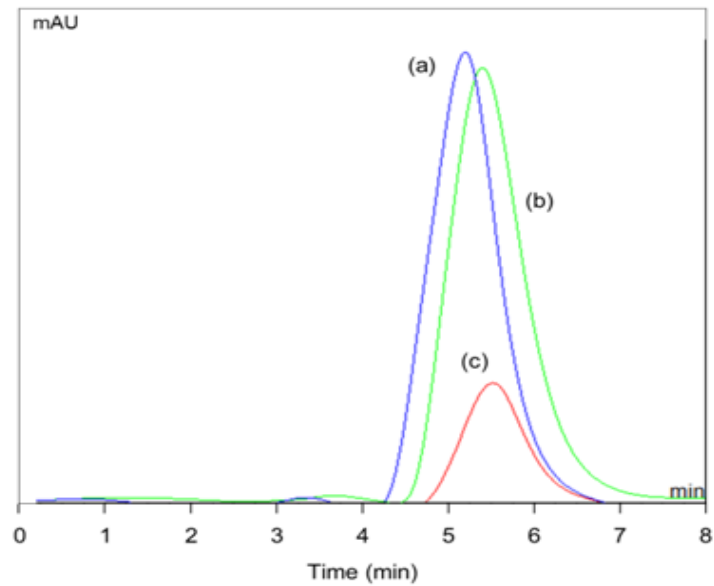

Fig. 7. Overlaid HPLC chromatograms of $5 \mathrm{ml}$ melamine standard of $100 \mathrm{mg} / \mathrm{l}$ : (a) before and after immersed with $0.02 \mathrm{~g}$ of (b) NIP (experiment 8) and (c) MIP (experiment 7) particles. 
increasing concentration of melamine as in experiment 9-12, all components were not completely miscible resulting in the formation of large amount of particle aggregation.

\section{CONCLUSION}

The uniform and stable sub-micrometer sized MIP particles representing high binding efficiency were successfully prepared by precipitation polymerization without any post treatment. The binding efficiencies of MIP using both cross-linkers (DVB and EGDMA) with melamine were approximately $20 \mathrm{mg} / \mathrm{g}-\mathrm{MIP}$. As large amount of MAA was included in the MIP particles, the purified MIP particles effectively redispersed in aqueous medium without coalescence which might be appropriate to be used as the stationary phase of solid phase extraction technique. In addition, the capability of all components being mixed and initiator content in precipitation polymerization effect on the binding property and MIP particle size that can be beneficial for the selection of the appropriate amount and type of functional monomer, cross-linker, template and initiator in the polymerization system.

\section{ACKNOWLEDGEMENT}

This work was supported by RMUTI research foundation scholarship in 2014 given to R.Karnka, P.Chaiyasat and A.Chaiyasat.

\section{REFERENCES}

1. Zhang, H.; Ye, L. and Mosbach, K. J. Mol. Recognit. 2006, 19(4), 248-259.

2. Salian, V.D.; Vaughan, A.D. and Byrne, M.E. J. Mol. Recognit . 2012, 25(6), 361-369.

3. Chen, L.; Xu, S. and Li, J. Chem. Soc. Rev. 2011, 40(5), 2922-2942.

4. Rezaei, B.; Mallakpour, S. and Majidi, N. Talanta. 2009, 78(2), 418-423.

5. Ara, B.; Chen, Z.; Shah, J.; Rasul Jan, M. and Ye, L. J. Appl. Polym. Sci. 2012, 126(1), 315321.

6. Torres, J.J.; Montejano, H.A. and Chesta, C.A. Macromol. Mater. Eng. 2012, 297(4), 342352.

7. Okutucu, B. and Önal, S. Talanta. 2011, 87, 74-79.

8. Sharma, P.S.; Pietrzyk-Le, A.; D'Souza, F. and Kutner, W. Anal. Bioanal. Chem. 2012, 402(10), 3177-3204.

9. Shi, Y.; Lv, H.; Lu, X.; Huang, Y.; Zhang, Y. and Xue, W. J. Mater. Chem. 2012, 22(9), 38893898.

10. Esfandyari-Manesh, M.; Javanbakht, M.; Atyabi, F. and Dinarvand, R. J. Appl. Polym. Sci. 2012, 125(3), 1804-1813.

11. Mohajeri, S.A.; Karimi, G.; Aghamohammadian, J. and Khansari, M.R. J. Appl. Polym. Sci. 2011, 121(6), 3590-3595.

12. Sun, H.; Lai, J.-P.; Chen, F. and Zhu, D.-R. Anal. Bioanal. Chem. 2015, 407(6), 17451752.
13. Ji, Y.; Yin, J.; Xu, Z.; Zhao, C.; Huang, H.; Zhang, H. and Wang, C. Anal. Bioanal. Chem. 2009, 395(4), 1125-1133.

14. Ugajin, H.; Oka, N.; Okamoto, T. and Kawaguchi, H. Colloid. Polym. Sci. 2013, 291(1), 109-115.

15. Dvorakova, G.; Haschick, R.; Chiad, K.; Klapper, M.; Müllen, K. and Biffis, A. Macromol. Rapid Comm. 2010, 31(23), 2035-2040.

16. Chen, Z. and Ye, L. J. Mol. Recognit. 2012, 25(6), 370-376.

17. Yoshimatsu, K.; Yamazaki, T.; Chronakis, I.S. and Ye, L. J. Appl. Polym. Sci. 2012, 124(2), 1249-1255.

18. Fitzhenry, L.; Manesiotis, P.; Duggan, P. and McLoughlin, P. Microchim. Acta. 2013, 180(15), 1421-1431.

19. Lu, X.-F.; Shi, Y.-f.; Lv, H.-L.; Fu, Y.-Y.; Ma, D. and Xue, W. J. Mater. Sci.-Mater. M. 2014, 25(6), 1461-1469.

20. Azenha, M.; Schillinger, E.; Sanmartin, E.; Regueiras, M.T.; Silva, F. and Sellergren, B. Anal. Chim. Acta. 2013, 802, 40-45.

21. Yu, P.; Sun, Q.; Li, J.; Tan, Z.; Yan, Y. and Li, C. J. Environ. Chem. Eng. 2015, 3(2), 797-806.

22. Oliveira, D.; Gomes, C.P.; Dias, R.C.S. and Costa, M.R.P.F.N. React. Funct. Polym. 2016, 107, 35-45.

23. Wulff, G. Angew. Chem. Int. Edit. 1995, 34(17), 1812-1832. 